\title{
Novel Rat Models for Atherosclerosis
}

\author{
Huaqing Chen ${ }^{*}$, Dali Li ${ }^{1}$, Mingyao Liu ${ }^{1,2 *}$
}

'Shanghai Key Laboratory of Regulatory Biology, Institute of Biomedical Sciences, School of Life Sciences, East China Normal University, Shanghai, China ${ }^{2}$ Institute of Biosciences and Technology, Department of Molecular and Cellular Medicine, Texas A\&M University Health Science Center, Houston, Texas, USA

\section{Article Info}

\section{Article Notes}

Received: April 08, 2018

Accepted: April 30, 2018

\section{*Correspondence:}

Dr. Huaqing Chen or Dr. Mingyao Liu, School of Life Sciences, East China Normal University, 500 Dongchuan Road, Shanghai 200241, China;

Telephone: +86-21-54344030;

E-mail: hqchen@bio.ecnu.edu.cn; myliu@bio.ecnu.edu.cn;

(c) 2018 Chen $\mathrm{H}$, Liu M. This article is distributed under the terms of the Creative Commons Attribution 4.0 International License.

\section{Key words:}

Apoe

Ldl receptor

Atherosclerosis

Rat model

Gene knockout

\section{ABSTRACT}

Mouse models have been widely used in atherosclerosis research and related therapy development. However, data suggested that it was difficult to translate discovery in mice to human. Thanks to the fast development and optimization in gene editing technology, atherosclerotic models in rats generated recently provide more options for different studies and could make a difference to the situation which is exclusively dependent on mouse. Here we reviewed novel rat models for atherosclerosis generated through genetic approaches in recent years, including Ldl receptor (LdIr) and apolipoprotein e (Apoe) knockout rats. The phenotypes in hyperlipidemia, atherosclerosis, as well as related inflammatory responses, were compared. These models developed typical atherosclerosis. More importantly, some of them present certain advantages over mouse models. These not only provide novel options for animal models of atherosclerosis, but also will benefit future investigations of atherosclerotic pathology and anti-atherosclerotic therapeutics.

\section{Introduction}

Atherosclerosis is not only the pathological basis, but also a pre-symptom of most cardiovascular diseases. Animal models of atherosclerosis have greatly increased our understanding of this disease. Among them, mouse models have been the most extensively used and provided invaluable data for the understanding of atherosclerosis initiation and development. However, the translation of discovery from mice to humans has been slow. Sometimes a drug which have been proved to be effective in mice was not effective in human. This problem seriously hindered the progress of drug development in atherosclerosis, mainly due to the difference in pathogenesis between mouse and human atherosclerosis ${ }^{1}$. Most severe clinical consequences of atherosclerosis in human usually arise from lesions in the coronary and cerebral arteries, whereas typical atherosclerosis in mice is induced by hypercholesterolemia and lesions mostly happen in the aorta root, aortic arch and proximal great vessels $\mathrm{s}^{2,3}$. Atherosclerosis in human is accompanied with intimal-thickening in early lesions and thick fibrous cap later, but these normally do not happen in mice ${ }^{4}$. Another important issue is, men usually have an earlier onset of clinical plaque burdens ${ }^{5,6}$, whereas in mouse, atherosclerosis phenotypes were more prominent in females ${ }^{7-9}$. In addition to the above discrepancy between mouse models and human disease, species-dependent effects might compromise the reliability of research data which were exclusively obtained from mouse models and this will also limit identification of unique features in human disease. Therefore, it is necessary 
to develop novel models for atherosclerosis in order to gain comprehensive understanding of development and promote further therapeutic studies of atherosclerosis.

Rat has been used as a model animal for over 100 years. Certain characteristics of lipid metabolism in rats are in-between those of humans and mice ${ }^{10}$. In addition, rat has advantages especially in studying cardiovascular diseases ${ }^{11,12}$. Compare to mouse models, rats have benefit as for easier in blood collection, dissection of blood vessels, etc. Furthermore, rats have a lower cost compared to larger animals like pigs and rabbits for purchasing, feeding and maintenance. However, rats are generally hypo-responsive to dietary cholesterol. Hyperlipidemia and atherogenesis were more easily induced by cholic acid or thiouracil containing high cholesterol and high fat diets ${ }^{13,14}$. Other treatments including chemical induction and mechanical trauma of the arteries have also been used to induce arterial lesions ${ }^{15}$. Sometimes combination of these strategies had to be used such as combining partial ligation of carotid artery with feeding a high fat diet ${ }^{16}$. Rats as animal models had one major disadvantages till recently, which is difficult in gene deletion using a gene targeting technology. Along with the fast development and optimization in gene editing technology, targeted gene deletion in rats has become easier and more common ${ }^{17,18}$. More and more labs are attempting to establish rat models to overcome certain limitations of mice, including our lab. In this review we mostly focus on novel rat models for atherosclerosis generated by genetic modification in recent years, and pay more attention to those models that are valuable for understanding atherosclerosis mechanism and evaluating anti-atherosclerotic treatments.

Both Ldl receptor (LDLR) and apolipoprotein E (ApoE) participate in the transport of cholesterol-rich lipoproteins. While Apoe deficiency in human is rare, $L d l r$ deficiency in human is related to hypercholesterolemia and the higher risk of atherosclerosis and coronary disease ${ }^{19,20}$. These patients develop arterial lesions which share some of the characteristics of the mouse lesions, including lesions in the aortic valves and aortic root. Both Apoe and Ldlr knockout (KO) mice have become the most used animal models of atherosclerosis since early $1990 \mathrm{~s}^{21,22}$. It was estimated that the proportion of atherosclerosis studies using these two models reached up to 80 percent of all the atherosclerosis studies using mouse models ${ }^{6}$. Here we will take a look at corresponding rat models first.

\section{Apoe deficient rat models}

Up to now, only three papers related to atherosclerosis in Apoe KO rats have been published. One reported occlusal disharmony accelerated atherosclerosis in Apoe KO rats, but only showed very early signs of lipid deposition and atherosclerosis ${ }^{23}$. Another Apoe $\mathrm{KO}$ rat was generated via the transcription activator-like effector nuclease (TALEN)mediated technology. It displayed a typical proatherogenic dyslipidemia when fed on a high-cholesterol diet, but failed to develop atherosclerotic plaques. The authors stated that the Apoe KO rat was different from Apoe KO mice as the rat was resistant to hyperlipidemia-induced inflammation and did not develop typical atherosclerosis. Instead it was found to develop atherosclerotic plaques in partially ligated carotid arteries ${ }^{24}$. Until this year, our lab became the first to report typical atherosclerosis development in CRISPR/Cas 9 generated Apoe KO rats. Our work showed that the Apoe KO rats we established in Sprague Dawley (SD) rats could effectively develop typical hyperlipidemia and plaques resemble disease in human ${ }^{18}$. The Apoe KO showed a decreased high-density lipoprotein cholesterol (HDL-c), but markedly upregulated total cholesterol (TC) and low-density lipoprotein cholesterol (LDL-c) levels. Hyperlipidemia induced by Apoe deficiency in our rats promoted endothelial inflammation by increasing atherosclerosis-related adhesive and inflammatory gene expression and led to typical atherosclerosis. The mutant developed primary atherosclerotic lesions even when fed on a normal diet, while Western diet dramatically exacerbated atherosclerosis. After 40 weeks on Western diet, the en face aortas of mutants showed a heavy plaque burden. After 64 weeks Western diet, more severe atherosclerotic plaques widely distributed throughout the arterial tree including the carotid arteries.

Since the above Apoe mutations were generated in SD rats, the differences might result from different deletion sites, analytical methods or observation time. For example the TALEN-Apoe KO carried a deletion of $14 \mathrm{bp}$ in exon 3 while in ours $130 \mathrm{bp}$ were deleted in exon 4 of Apoe gene. In addition, they only observed up to 18-20 weeks of age which might be too short. Results from our Apoe KO rat support the notion that atherosclerosis is developed mainly from hyperlipidemia ${ }^{25}$, and differ from the previous suggestion that rat could not develop typical atherosclerosis. The CRISPR/Cas9 Apoe KO rat provided a suitable option for atherosclerosis research, and it has the benefit that the lesions in males were slightly heavier than that in females, not the opposite as in mouse models. In addition, these mutant rats developed atherosclerotic lesions when fed on normal diet which caused a less extreme lipid alteration. Thus CRISPR/Cas9 Apoe KO rat model could be suitable in evaluating regulatory effects in atherosclerosis of certain genes with no impact on lipid metabolism ${ }^{26}$.

\section{Ldlr deficient rat models}

The first functional knockout rat (F344/Slc) for Ldlr was reported in 2012 as a result of N-ethyl-N-nitrosourea induced missense mutation in exon 4.The homozygous mutant fed a normal diet showed higher levels of TC and LDL-c. When fed a Western diet, the mutant rats exhibited 
severe hyperlipidemia and significant lipid deposition all over the aortas. Females displayed more severe atherosclerotic lesions than the males which was the same as in mouse models, but opposite to human ${ }^{27}$. Then a paper in 2016 reported the generation of a Ldlr KO rat with a 19 bp deletion in exon 7 via Zinc-finger nuclease (ZFN) technology. There was elevated TC, TG, LDL-c and VLDL-c in the plasma of the mutant, although no alteration in blood glucose and free fatty acids. The authors stated it did not develop arterial plaques when fed on a normal diet, although no related data was presented ${ }^{28}$. Another Ldlr KO rat generated in 2017 also by ZFN was reported to develop obesity and atherosclerotic plaques when fed on a Western diet ${ }^{29}$. Using the CRISPR/Cas9 technique, we generated Ldlr KO rat model in SD background. The mutant rats developed critical features presented in human atherosclerosis, including marked hyperlipidemia, different stages of atherosclerotic lesions and liver steatosis. The plasma lipoprotein profile from mutant rats maintained on normal diet showed elevated cholesterol in the VLDL fraction. Western diet greatly aggravated dyslipidemia, inflammation and atherosclerosis. After 40 weeks on Western diet, the en face aortas of the mutant rats displayed heavy plaques.

The characteristics of hyperlipidemia and atherosclerosis were quite similar between the ZFN-Ldlr KO generated in 2017 and our Ldlr KO rats. However, there were still some distinctive differences. While HDL in our Ldlr KO rats was increased as compared to that in WT rats, the level in ZFN-LdIr KO was decreased. Also, while no significant lesion was found in the ZFN-Ldlr KO rat on a normal diet, we observed early lesions in normal diet fed CRISPR/Cas9 Ldlr KO rats. This is probably associated with the higher inflammatory responses due to LRPs deficiency which was not found in the ZFN- Ldlr KO model. In addition, the ZFN-Ldlr KO rat was obese and had glucose intolerance, which was a surprise even for the authors ${ }^{29}$. Both of these phenotypes were not observed in our Ldlr KO rats. In fact, there was no change in body weight and glucose tolerance in Ldlr $\mathrm{KO}$ mice $\mathrm{e}^{30,31}$, also not all patients with familial hypercholesterolemia are obese or glucose intolerance. These two Ldlr deletion mutants were both generated in SD background, and used similar diets. However, there was a moderate difference in deletion sites. While the deletion in our mutant was $118 \mathrm{bp}$ in exon 4, in the ZFN-Ldlr KO rat it was $337 \mathrm{bp}$ at the junction of intron 3 and exon 4 . It is tempting to speculate that certain regulatory element exists in intron 3.

\section{Other genetically modified rat models}

\section{Apoe/Ldlr double KO rat}

By cross-breeding Apoe KO and Ldlr KO rat, we also generated double $\mathrm{KO}$ rats and investigated their phenotypic characteristics in hyperlipidemia and atherosclerosis. Most of the phenotypes were similar to both of the single KO rats, including a severe hyperlipidemia and redistribution of cholesterol in different lipoprotein fractions. Western diet also dramatically exacerbated hyperlipidemia and atherosclerosis. 40-weeks feeding on a Western diet led to a heavy burden of lesions in aortas of the mutants. After 64 weeks on the Western diet, the lesion area in aortas reached up to 30 percent. However, the most interesting point was that the double KO displayed a markedly higher atherosclerotic plaques in males than in females ${ }^{18}$, similar to the profile in human disease ${ }^{5,6}$. In addition, although no significant difference was found between the double KO and single KO rats, it seems macrophage infiltration was more prominent in the double $\mathrm{KO}$ rats $^{18}$.

\section{JCR:LA- $c p$ strain}

The JCR:LA- $c p$ strain was established from the original Koletsky colony, a mutation in a rat line originated from a cross between SD and spontaneously hypertensive rats ${ }^{32}$. It has a spontaneous mutation of $c p$, maintaining insulinresistant and atherosclerosis-prone characteristics. Studies by both scanning and transmission electron microscopy exhibited extensive atherosclerotic plaques all over the arterial system of male mutants ${ }^{33}$. The lesions resemble human atherosclerosis.

\section{Human CETP transgenic rat}

Although transgenic rats were generated since the $1990 \mathrm{~s}^{34}$, few transgenic rats have been shown to produce atherosclerosis. However, a rat overexpressing human cholesteryl ester transfer protein (CETP) in a Dahl saltsensitive hypertensive strain did produce atherosclerosis ${ }^{35}$. It was reported that the male rats fed normal diet showed age-dependent hyperlipidemia and atherosclerotic lesions. More importantly, they also developed myocardial infarctions and showed decreased survival. These rats showed quite different phenotypes from various mouse atherosclerosis models, indicating the necessity for complex disease modeling in different animals. It has to be kept in mind though, that there is some limitation for transgenic animals as gene integration might occur at random sites and the transgene could present multiple copies of the specific gene.

\section{Conclusions}

Atherosclerosis is a complicated disease which proceeds at different paces at different situations and blood vessels. Although useful and convenient, the present mouse models clearly are not enough for in depth investigation of atherosclerosis initiation and consequences. So, new animal models are needed for the discovery of optimal treatment of this chronic disease. The growing number of rat models is part of the effort in this direction. 
As a chronic inflammatory disease, various cell populations participate in development of atherosclerosis. Activated endothelial cells express adhesion molecules that attract blood cells into the vessel wall. Monocytes are among the most presented cells migrating into the intima. They differentiate into macrophages and present antigens to activate other immune cells through secretion of a variety of cytokine and growth factors ${ }^{36}$. The $L d l r$ and Apoe single or double KO rats obtained in our lab exhibited significant inflammation in the initiation with the mononuclear cell infiltration in the aortic intima and macrophage accumulation in the later stage of lesions, with more prominent macrophages presented in double $\mathrm{KO}$ as compared to single $\mathrm{KO}$ rats. Thus in these rat models immune responses were directly linked to the metabolic disturbance and pathogenesis of atherosclerosis, mimicking the situation in human. They would be useful in the investigation of inflammation, lipid deposition and different stages of atherosclerosis.

In humans, men usually develop earlier and heavier atherosclerotic plaques than women ${ }^{5,6}$, which is not manifested in mouse models. Among rat models, the F344/ Slc Ldlr KO displayed heavier atherosclerotic lesions in females than males, which is opposite to the human disease ${ }^{27}$. No related data was provided in JCR:LA- $c p$ strain and CETP transgenic rats, although males were used in the reports $^{33,35}$. Interestingly, CRISPR/Cas9 generated mutant rats especially the Apoe/Ldlr double KO displayed a sex difference profile more similar to human, in which males had heavier plaque burdens than females ${ }^{18}$. These models will greatly benefit future development of more powerful therapeutics for this widespread disease.

It is foreseeable that more and more rat models with defined genetic makeup will be generated thanks to the growing power of genetic editing and the sequencing of rat genome. Rat model will become an excellent option for atherosclerosis and related studies. The increased availability and utilization of rat models generated by genetic manipulation will greatly add to the wealth of experimental data in the understanding of human translational investigations, such as the elucidation of the detailed mechanism and of action of clinical treatments.

\section{Acknowledgments}

This work was supported by grant funding from the National Natural Science Foundation of China [81330049 to M.L.], and Innovation Program of Shanghai Municipal Education Commission [2017-01-07-00-05-E00011 to M.L.].

\section{References}

1. Bentzon, JF, Falk E. Atherosclerotic lesions in mouse and man: is it the same disease. Curr Opin Lipidol. 2010; 21: 434-440.

2. Getz GS, Reardon CA. Animal models of atherosclerosis. Arterioscler Thromb Vasc Biol. 2012; 32: 1104-1115.
3. Libby, P, Inflammation in atherosclerosis, Nature, 2002;420:868-874.

4. Schwartz SM, deBlois D, O'Brien ER. The intima. Soil for atherosclerosis and restenosis. Circ Res. 1995; 77: 445-465.

5. Wang Z, Klipfell E, Bennett BJ, et al. Gut flora metabolism of phosphatidylcholine promotes cardiovascular disease. Nature. 2011; 472: $57-63$

6. von Scheidt M, Zhao Y, Kurt Z, et al. Applications and Limitations of Mouse Models for Understanding Human Atherosclerosis. Cell Metab. 2017; 25: 248-261.

7. Caligiuri G, Nicoletti A, Zhou X, et al, Effects of sex and age on atherosclerosis and autoimmunity in apoE-deficient mice. Atherosclerosis. 1999; 145: 301-308.

8. Teupser D, Persky AD, Breslow JL. Induction of atherosclerosis by lowfat, semisynthetic diets in LDL receptor-deficient C57BL/6J and FVB/ NJ mice: comparison of lesions of the aortic root, brachiocephalic artery, and whole aorta (en face measurement). Arterioscler Thromb Vasc Biol. 2003; 23: 1907-1913.

9. Teupser D, Pavlides S, Tan M, et al. Major reduction of atherosclerosis in fractalkine (CX3CL1)-deficient mice is at the brachiocephalic artery, not the aortic root. Proc Natl Acad Sci U S A. 2004; 101: 17795-17800.

10. Heinonen SE, Genove G, Bengtsson E, et al. Animal models of diabetic macrovascular complications: key players in the development of new therapeutic approaches. J Diabetes Res. 2015; 2015: 404085.

11. Iannaccone PM Jacob HJ. Rats!, Dis Model Mech. 2009; 2: 206-210.

12. Lee N. Physiogenomic strategies and resources to associate genes with rat models of heart, lung and blood disorders. Exp Physiol. 2007; 92: 992-1002.

13. Joris I, Zand T, Nunnari JJ, et al. Studies on the pathogenesis of atherosclerosis. I. Adhesion and emigration of mononuclear cells in the aorta of hypercholesterolemic rats. Am J Pathol. 1983; 113: 341358.

14. Fu R, Zhang Y, Guo Y, et al. Digital gene expression analysis of the pathogenesis and therapeutic mechanisms of ligustrazine and puerarin in rat atherosclerosis. Gene. 2014; 552: 75-80.

15. Ritskes-Hoitinga J, Beynen AC. Atherosclerosis in the rat. Artery. 1988; 16: 25-50.

16. You Y, Liu W, Li Y, et al. Joint preventive effects of swimming and Shenlian extract on rat atherosclerosis. Clin Hemorheol Microcirc. 2011; 47: 187-198.

17. You P, Hu H, Chen Y, et al. Effects of Melanocortin 3 and 4 Receptor Deficiency on Energy Homeostasis in Rats. Sci Rep. 2016; 6: 34938.

18. Zhao Y, Yang $Y$, Xing R, et al. Hyperlipidemia induces typical atherosclerosis development in Ldlr and Apoe deficient rats. Atherosclerosis. 2018; 271: 26-35.

19. Abul-Husn NS, Manickam K, Jones LK, et al. Genetic identification of familial hypercholesterolemia within a single U.S. health care system. Science. 2016; 354.

20. Schaefer EJ, Gregg RE, Ghiselli G, et al. Familial apolipoprotein E deficiency. J Clin Invest. 1986; 78: 1206-1219.

21. Ishibashi S, Goldstein JL, Brown MS, et al. Massive xanthomatosis and atherosclerosis in cholesterol-fed low density lipoprotein receptornegative mice. J Clin Invest. 1994; 93: 1885-1893.

22. Plump AS, Smith JD, Hayek T, et al. Severe hypercholesterolemia and atherosclerosis in apolipoprotein E-deficient mice created by homologous recombination in ES cells. Cell. 1992; 71: 343-353.

23. Ekuni D, Yoneda T, Endo Y, et al. Occlusal disharmony accelerates the initiation of atherosclerosis in apoE knockout rats. Lipids Health Dis. 2014; 13: 144. 
24. Wei S, Zhang Y, Su L, et al. Apolipoprotein E-deficient rats develop atherosclerotic plaques in partially ligated carotid arteries. Atherosclerosis. 2015; 243: 589-592.

25. Ross R. The pathogenesis of atherosclerosis--an update. N Engl J Med. 1986; 314: 488-500.

26. Stylianou IM, Bauer RC, Reilly MP, et al. Genetic basis of atherosclerosis: insights from mice and humans. Circ Res. 2012; 110: 337-355.

27. Asahina M, Mashimo T, Takeyama M, et al. Hypercholesterolemia and atherosclerosis in low density lipoprotein receptor mutant rats. Biochem Biophys Res Commun. 2012; 418: 553-558.

28. Wang HY, Quan C, Hu C, et al. A lipidomics study reveals hepatic lipid signatures associating with deficiency of the LDL receptor in a rat model. Biol Open. 2016; 5: 979-986.

29. Sithu SD, Malovichko MV, Riggs KA, et al. Atherogenesis and metabolic dysregulation in LDL receptor-knockout rats. JCI Insight. 2017; 2.

30. Pauta M, Rotllan N, Vales F, et al. Impaired liver regeneration in Ldlr-/- mice is associated with an altered hepatic profile of cytokines, growth factors, and lipids. J Hepatol. 2013; 59: 731-737.

31. Ellis A, Cheng ZJ, Li Y, et al. Effects of a Western diet versus high glucose on endothelium-dependent relaxation in murine micro- and macro-vasculature. Eur J Pharmacol. 2008; 601: 111-117.

32. Koletsky S. Pathologic findings and laboratory data in a new strain of obese hypertensive rats. Am J Pathol. 1975; 80: 129-142.

33. Richardson M, Schmidt AM, Graham SE, et al. Vasculopathy and insulin resistance in the JCR:LA-cp rat. Atherosclerosis. 1998; 138: 135-146.

34. Mullins JJ, Peters J, Ganten D. Fulminant hypertension in transgenic rats harbouring the mouse Ren-2 gene. Nature. 1990; 344: 541-544.

35. Herrera VL, Makrides SC, Xie HX, et al. Spontaneous combined hyperlipidemia, coronary heart disease and decreased survival in Dahl salt-sensitive hypertensive rats transgenic for human cholesteryl ester transfer protein. Nat Med. 1999; 5: 1383-1389.

36. Ait-Oufella H, Taleb S, Mallat Z, et al. Recent advances on the role of cytokines in atherosclerosis. Arterioscler Thromb Vasc Biol. 2011; 31: 969-979. 